\title{
Neutron rich nuclei in density dependent relativistic Hartree-Fock theory with isovector mesons
}

\author{
B. Q. Chen, Z. Y. Ma ${ }^{\dagger}$ \\ China Institute of Atomic Energy, Beijing 102413, P.R.China \\ F.Grümmer ${ }^{\dagger}$ and S. Krewald \\ Institut für Kernphysik, Forschungszentrum Jülich $\mathrm{GmbH}$ \\ D-52425 Jülich, Germany
}

March 24, 2021

\begin{abstract}
Density dependent relativistic Hartree-Fock theory has been extended to describe properties of exotic nuclei. The effects of Fock exchange terms and of $\pi$ - and $\rho$ - meson contributions are discussed. These effects are found to be more important for neutron rich nuclei than for nuclei near the valley of stability.
\end{abstract}

PACS: 21.10.Dr, 21.10.Gv, 21.60.Jz, 24.10.Jv

Keywords: relativistic Hartree-Fock, neutron rich nuclei, Ca isotopes

Among the various theoretical models for the bulk properties of nuclei, the relativistic mean field theory (RMF) has been particularly successful [1, 2], 3]. Relativistic mean field theory can be regarded as an effective field theory which

${ }^{*}$ Also: Center of Nuclear Theoretical Physics, National Laboratory of Heavy Ion Accelarator, Lazhou 730000, P.R.China

${ }^{\dagger}$ Also: Institute of Theoretical Physics, Beijing 100080, P.R.China

${ }^{\ddagger}$ corresponding author: Tel: +49 (2461) 61 2802, Fax: +49 (2461) 61 3930, E-mail: F.Gruemmer@fz-juelich.de 
achieves a description of nuclear properties throughout the entire periodic table with high precision. It is based on a relativistic Lagrangian which includes the nucleon, the omega-, the sigma-, and the rho-meson (which reproduces the asymmetry energy of nuclear matter) as well as non-linear self-interactions of scalar mesons. The inclusion of the non-linear self-interactions of the sigma field introduces density-dependent interactions in the model, which are crucial to reproduce quantitatively the bulk properties of nuclear matter and finite nuclei. Sharma et al. [4], Lalazissis et al. [5], Maharana et al. [6], Hirata el al. [7] and Lalazissis et al. [8] have systematically investigated the binding energies, deformations, rms radii, one (or two) nucleon separation energies, quadrupole moments and isotopic shifts of nuclei far from the stability line. A review of the relativistic mean field model has been given by Ring [9].

Relativistic effects have been found to be important for theories starting from the realistic nucleon-nucleon interaction, too. The Dirac- Brueckner-HartreeFock(DBHF) approach based on the meson-exchange potential Bonn A successfully reproduces nuclear matter saturation properties [10], while non-relativistic models failed. The DBHF approach has been applied to finite nuclei by Muether et al. [11]. Due to the complexity of the DBHF in finite nuclei, only light spherical nuclei have been investigated.

Brockmann and Toki have suggested a method that allows to link the binding energies and radii of finite nuclei to the nucleon-nucleon interaction via a local density dependence approximation to the DBHF [12], the "relativistic density dependent Hartree" model(RDH). In this approach, density dependent coupling constants of the meson-nucleon interactions are derived from DBHF calculations in nuclear matter. Those coupling constants are used in a relativistic Hartree calculation performed for finite nuclei. The model therefore is parameter free in the sense that no parameters are adjusted to data of the nuclear many-body problem. It does, however, contain some implicit assumptions. One assumes that it is sufficient to include density dependent modifications of the coupling constants of the sigmaand the omega-meson only. This assumption may be justified by the very success of the relativistic mean field model which shows that the sigma- and the omegameson dominate the saturation properties of nuclei, as is obvious in closed-shell nuclei and in the Hartree approximation. Moreover, the Dirac Brueckner HartreeFock calculation provides a scalar and a vector contribution to the self-energy of the nucleon for each density. These are two constraints which can be used to determine two parameters of the effective Lagrangian. It is then natural to adjust the coupling constants of the two most relevant mesons to those numbers.

The RDH approach with an effective sigma, omega, and rho meson gives satisfactory descriptions of ground state properties of a few stable nuclei [12], although the fit quality cannot match the one achieved in the RMF theories. Investigations of this type are very important for our understanding of properties of finite nuclei 
in a parameter free way. The model so far has been applied only to a relatively small number of nuclei. Therefore it is of great interest to extend such density dependent relativistic model to deformed and exotic nuclei. Recently, the RDH has been applied to deformed nuclei and the investigations show large deviations from the experimental data for the quadrupole moments of rare-earth nuclei [13].

In the RDH approach, Pauli exchange effects have been neglected. Fritz, Müther and Machleidt [14] pointed out, however, that the nuclear radii are reduced once the Pauli exchange effects are taken into account. On the other hand, after incorporating both pion and rho-exchange (including in particular the tensor part of the rho-nucleon coupling) into the relativistic density dependent HartreeFock approach, larger radii are found [15]. The findings of [15] suggest that the effects of Fock terms be investigated more thoroughly. In the case of spin-orbit splitting, the inclusion of the Fock terms appears to be essential. In ${ }^{48} \mathrm{Ca}$, relativistic mean field theory gives a splitting of the $d_{5 / 2}$ and the $d_{3 / 2}$ levels of 9.32 $\mathrm{MeV}$, whereas the relativistic density dependent Hartree-Fock theory gives 3.19 $\mathrm{MeV}$ which comes closer to the experimental value of $4.3 \mathrm{MeV}$ [15].

Another place where the influence of Fock terms may be manifest is nuclei with a large neutron excess. The masses and the density distributions of neutronrich nuclei are important to predict the abundance of elements [16]. Systematic experimental investigations of exotic nuclei have become possible because of the recent advent of radioactive beam facilities [17].

In a study of neutron-rich nuclei, one has to include both the pion and the rhomeson. This requires to consider the effects of short-range correlations. Recently, Marcos et al. [18] have developed a nuclear matter model of the two-body correlation function. It provides a physical way to deal with the zero-range piece of $\pi$ and $\rho$ interaction in the medium. In our investigation, this effect of short-range correlations is incorporated by removing contact interactions in $\pi$ and $\rho$ exchanges [19]. In addition, Marcos et al. show that the effects of short range correlations can be simulated by modifiying the meson masses in the medium, at least in the Hartree approximation and for vanishing momentum transfer.

In this letter, we apply the relativistic density dependent Hartree (RDH) and Hartree-Fock (RDHF) to stable as well as neutron rich nuclei. We extend the calculation to include the pion and the rho-meson using the following interaction Lagrangian:

$$
\begin{aligned}
\mathcal{L}_{\mathcal{I}}= & \bar{\psi}\left(g_{\sigma} \sigma-g_{\omega} \gamma^{\mu} \omega_{\mu}-g_{\rho} \gamma^{\mu} \rho_{\mu} \cdot \tau\right) \psi \\
& +\frac{f_{\rho}}{2 M} \bar{\psi} \sigma^{\mu \nu} \partial_{\mu} \rho_{\nu} \cdot \tau \psi \\
& -\bar{\psi} e \gamma^{\mu} A_{\mu} \frac{1}{2}\left(1+\tau^{3}\right) \psi-\frac{f_{\pi}}{m_{\pi}} \bar{\psi} \gamma_{5} \gamma^{\mu} \partial_{\mu} \pi \cdot \tau \psi
\end{aligned}
$$

where $\tau_{i}$ indicate the isospin Pauli matrices. The effective strengths of couplings 


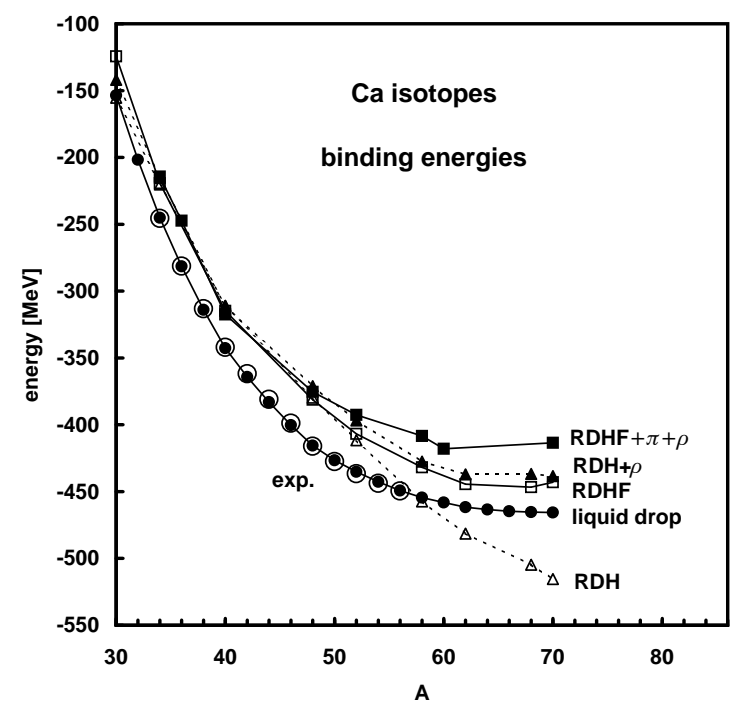

Figure 1: The binding energies of the Ca isotopes are shown. The open circles represent the experimental data [23] and the full line with the filled circles show a fit using a liquid drop model [24]. The RDH calculation including the rho meson( dashed line with filled triangles ), the RDHF model with sigma and omega mesons only ( full line with open squares ), and the RDHF model including the isovector mesons ( full line with filled squares) are compared. An RDH calculation without isovector mesons (dashed line with open triangles ) is given as a reference point for the RDHF calculations.

between the nucleon and mesons are denoted by the coupling constants $g_{i}$ or $f_{i}$ ( $i=\sigma, \omega, \rho, \pi$ ), respectively. The formalisms of the RDH and RDHF have been presented in detail in Refs. [14, 21, 15, 19, 22].

As Lenske pointed out rearrangement terms have to be included once density dependent coupling constants are used [20]. The inclusion of the rearrangement terms in the RDH slightly changes the binding energy, but improves the rootmean-square radii and density distributions. For simplicity, the rearrangement terms have not been included in this letter, since we are interested in the contributions of the Fock term and isovector mesons, pion and rho in neutron-rich nuclei qualitatively.

For the effective interaction, the coupling constants of the sigma- and omegamesons are adjusted to reproduce the scalar and vector potentials $\mathrm{U}_{s}$ and $\mathrm{U}_{o}$ in nuclear matter of the DBHF results using the Bonn A potential [10]. The density dependence of the coupling constants is then adjusted to reproduce the nucleon self-energies at each density in the cases of RMF or RHF with or without isovector 


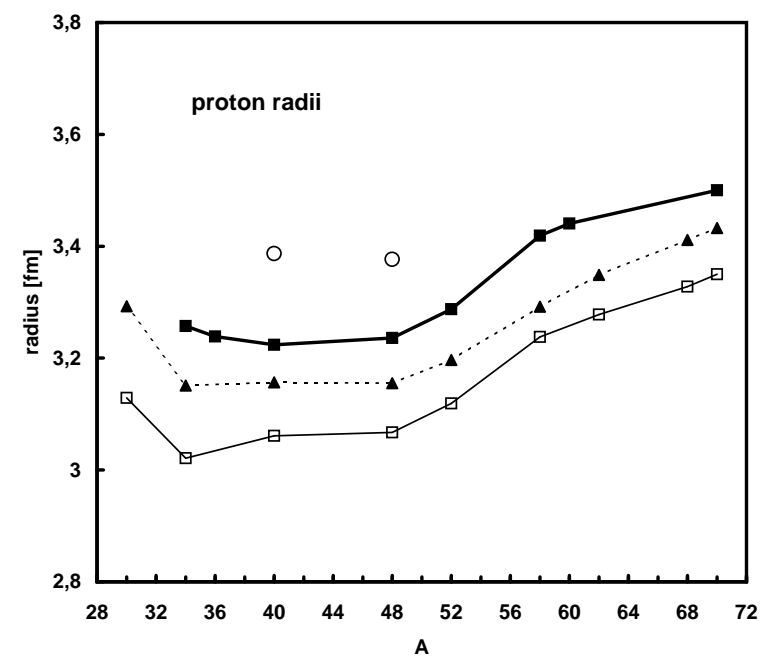

Figure 2: The proton radii of the Ca isotopes are shown for some of the models of Fig.1. The calculations are denoted by the same symbols as in Fig.1. In addition the open circles show the experimental proton radii of ${ }^{4} 0 \mathrm{Ca}$ and ${ }^{7} 0 \mathrm{Ca}$.

mesons. The nucleon and $\omega$ meson masses are chosen to be the same as in the DBHF calculation, where $M=938.9 \mathrm{MeV}, m_{\sigma}=550 \mathrm{MeV}$ and $m_{\omega}=782.6$ $\mathrm{MeV}$. For the $\pi \mathrm{NN}$ vertex pseudo-vector coupling and for $\rho \mathrm{NN}$ vector and tensor couplings are used. The masses and coupling constants of isovector mesons are fixed to be $\mathrm{m}_{\pi}=138 \mathrm{MeV}, \mathrm{m}_{\rho}=770 \mathrm{MeV}, \frac{f_{\pi}^{2}}{4 \pi}=0.08, \frac{g_{\rho}^{2}}{4 \pi}=0.55$ and $\frac{f_{\rho}}{g_{\rho}}=3.7$. In the RDHF calculation with isovector mesons, the sigma meson mass has been adjusted to $m_{\sigma}=450 \mathrm{MeV}$ ( after removal of the contact terms of the pion and the rho meson ). The RDHF calculation without isovector mesons has been performed with $m_{\sigma}=550 \mathrm{MeV}$.

Our calculations are based on the one-boson exchange potential Bonn A [10], which is used as an input for a Dirac Brueckner Hartree-Fock calculation. We will study the properties of exotic nuclei using three different approaches:

1. Relativistic density dependent Hartree theory $(\mathrm{RDH})$ with the $\sigma, \omega$, and $\rho$ mesons.

2. Relativistic density dependent Hartree-Fock theory (RDHF) with $\sigma$ and $\omega$ mesons only.

3. Relativistic density dependent Hartree-Fock theory with $\sigma, \omega, \pi$ and $\rho$ mesons $(\mathrm{RDHF}+\pi+\rho)$. 


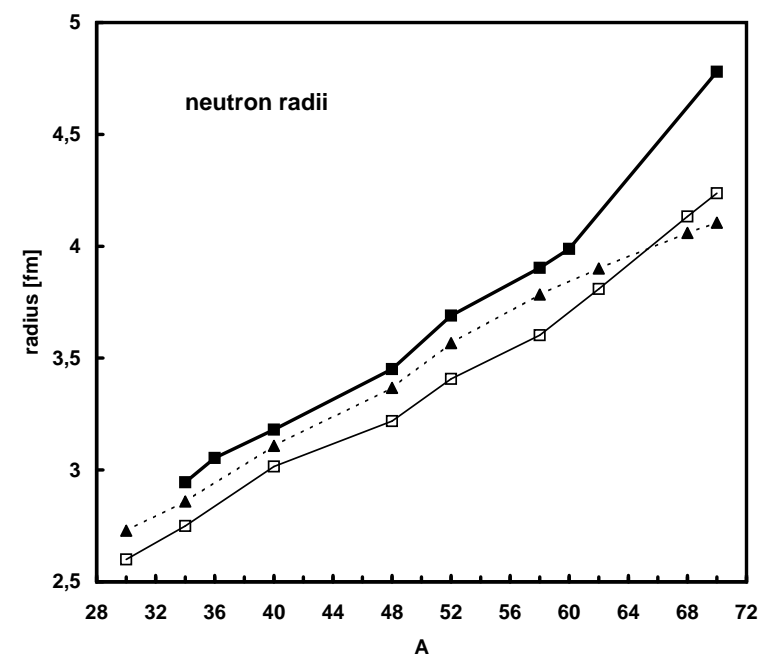

Figure 3: The neutron radii of the Ca isotopes are shown for some of the models of Fig.1. The calculations are denoted by the same symbols as in Fig.1.

In Fig.1, we show the binding energies of the $\mathrm{Ca}$ isotopes obtained in our model. Experimentally, the binding energies of the $\mathrm{Ca}$ isotopes are known only till $A=56$ (open circles ) [23]. We use the finite range liquid drop model by Möller, Nix and Kratz [24] to extrapolate the binding energies of the exotic nuclei till $A=70$. The RDH approach allows to obtain a fair agreement with the experimental data, if one incorporates the rho meson with a coupling constant $\left(\frac{g_{\rho}^{2}}{4 \pi}=0.978\right)$ that has been chosen to reproduce the asymmetry energy.

In both the relativistic density dependent Hartree- and Hartree-Fock- approximation, the omega-nucleon and the sigma-nucleon coupling constants are adjusted to reproduce the nucleon self energy in nuclear matter. Therefore it is not too surprising that both methods reproduce the binding energies of $\mathrm{N}=\mathrm{Z}$ nuclei with comparable accuracy. Indeed, Fritz et al. find in ${ }^{40} \mathrm{Ca}$ a binding energy per particle of $E / A=-8.21 \mathrm{MeV}$ in the RDH approach, while the RDHF calculation yields $E / A=-7.76 \mathrm{MeV}$ which is in fair agreement with the experimental value of $-8.50 \mathrm{MeV}$. The inclusion of the pion did not change the radius of ${ }^{40} \mathrm{Ca}$, but slightly increased the binding energy [14]].

With increasing neutron excess, however, the inclusion of the Fock terms leads to a reduction of the binding energy. The inclusion of the pion and the rho leads to further decreases of the binding energies for the exotic nuclei. The comparison with the experimental data shows that all approaches: $\mathrm{RDH}$ with proper rho coupling and RDHF with and without isovector mesons could reproduce the slope of 


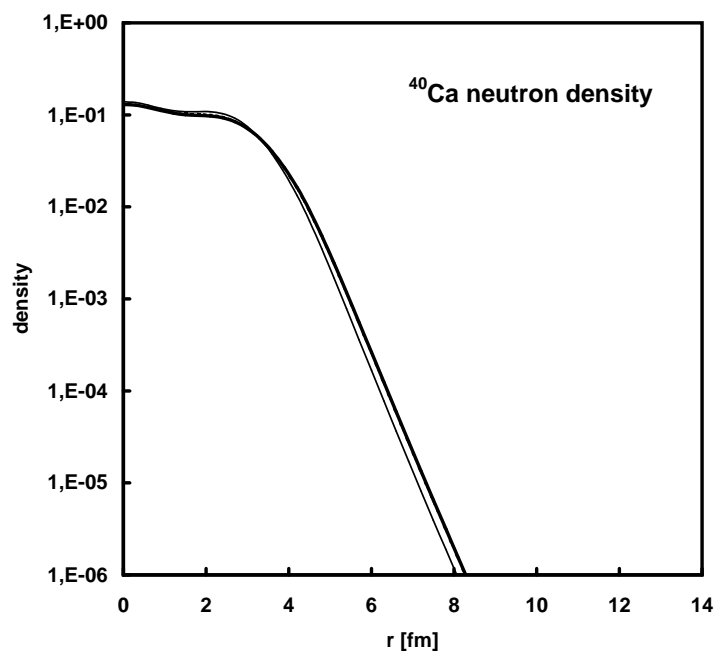

Figure 4: Neutron densities for ${ }^{40} \mathrm{Ca}$ : thin solid line: RDHF with $\omega$ and $\sigma$ only; thick solid line RDHF including isovector mesons; dashed line (hidden beneath the thick solid line) : RDH with $\rho$.

the curve of the experimental binding energies. At the RDHF level, the coupling constant of the rho-meson is not adjusted to the asymmetry energy, but taken from the underlying two-body interaction.

The proton radii are displayed in Fig. 2a. In comparison with a RDH calculation ( including the rho meson), the Fock term reduces the proton radii for all nuclei of the isotope chain. But the inclusion of the pion and in particular the rhomeson in the RDHF calculation increases the proton radii. For the neutron radii ( Fig.2b ), a reduction due to the Fock term is found for mass numbers less than $A=66$, while for the extremely neutron rich nuclei, one even finds an increase of the neutron radii. The inclusion of the pion and the rho leads to an overall increase of the neutron radii.

The various effects can be very clearly seen in the density distributions. We show here as examples the neutron density distributions for ${ }^{40} \mathrm{Ca}$ (Fig. 3a) and ${ }^{70} \mathrm{Ca}$ (Fig. 3b). We see that in the case of ${ }^{40} \mathrm{Ca}$ neither the Fock terms nor the inclusion of isovector mesons change the neutron density distribution considerably, so that one may argue in this case that the RDH description is sufficiently good. On the other hand we see strong effects of the Fock terms as well as the isovector mesons in the case of ${ }^{70} \mathrm{Ca}$. This indicates that especially in the case of very neutron rich nuclei, where e.g. a neutron halo is expected, one has to take into account all additional effects. To conclude, the density dependent relativistic 


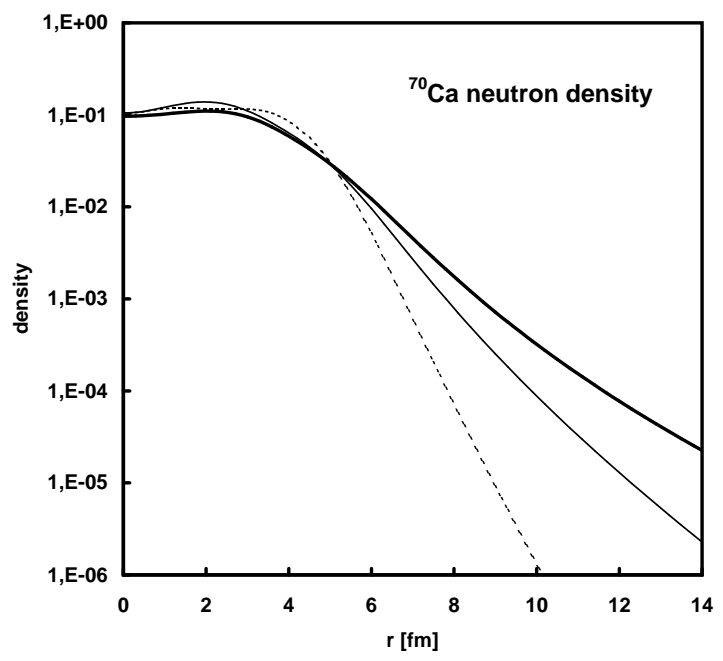

Figure 5: Neutron densities for ${ }^{70} \mathrm{Ca}$ : thick solid line: RDHF including isovector mesons; thin solid line: RDHF with $\omega$ and $\sigma$ only; dashed line: $R D H$ with $\rho$.

Hartree-Fock theory has been employed to investigate properties of neutron rich nuclei. For the first time the effect of Fock exchange terms and isovector mesons on these properties has been studied. It is found that these terms give large contributions to properties of exotic nuclei.

This work was supported by the Deutsche Forschungsgemeinschaft (DFG) and the China National Natural Science Foundation (CNNSF). We thank Professor J. Speth for constant support and helpful discussions. B.Q. Chen and Z.Y. Ma would like to thank for the hospitality of the Institut für Kernphysik, Forschungszentrum Jülich during their visit.

\section{References}

[1] B.D. Serot and J.D. Walecka, Adv. Nucl. Phys. 16 (1986) 1, Recent progress in quantum hadrodynamics, nucl-th/9701058

[2] P.G. Reinhard, Rep. Prog. Phys. 52 (1989) 439.

[3] Y.K. Gambhir, P. Ring and A. Thimet, Ann. Phys. 198 (1990) 132.

[4] M.M. Sharma, G.A. Lalazissis, W. Hillebrandt and P. Ring, Phys. Rev. Lett. 72 (1994) 1431. 
[5] G.A. Lalazissis and M.M. Sharma, Nucl. Phys. A586 (1995) 201.

[6] J.P. Maharana, Latha S. Warrier and Y. K. Gambhir, Ann. Phys. 250 (1996) 237.

[7] D. Hirata, H. Toki and I. Tanihata, Nucl. Phys. A589 (1995) 239.

[8] G.A. Lalazissis, A.R. Farhan and M.M. Sharma, Light nuclei near neutron and proton drip lines in the relativistic mean-field theory, hucl-th/9711038.

[9] P. Ring, Prog. Part. Nucl. Phys. 37 (1996) 193.

[10] R.Brockmann and R.Machleidt, Phys. Rev. C42 (1990) 1965.

[11] H.Muether, R.Machleidt and R.Brockmann, Phys. Rev. C42 (1990) 1981.

[12] R. Brockmann and H. Toki, Phys. Rev. Lett. 68 (1992) 3408

[13] M.L.Cescato and P.Ring, Phys. Rev. C57 (1998)134.

[14] R. Fritz, H. Müther and R. Machleidt, Phys. Rev. Lett. 71 (1993) 46; Phys. Rev. C49 (1994) 633.

[15] Z.Y. Ma, H.L. Shi and B.Q. Chen, Phys. Rev. C50 (1994) 3170; C52 (1995) 144.

[16] F. Thielemann et al., Nucl. Phys. A670 (1994) 329.

[17] Proceedings of the Fourth international conference on radioactive nuclear beam, 3-7 June 1996, Edited by S. Kubono, T. Kobayashi and I. Tanihata Nucl. Phys. A616 (1997) Nos. 1,2.

[18] S. Marcos, J.-F. Mathiot, M.Lopez-Quelle, R.Niembro, P.Bernardos, Nucl.Phys. A600 (1996) 529.

[19] A. Bouyssy, J.F. Mathiot, N. Van Giai and S. Marcos, Phys. Rev. C36 (1987) 380.

[20] H. Lenske and C. Fuchs, Phys. Lett. B345 (1995) 355.

[21] H.F. Boersma and R. Malfliet, Phys. Rev. C49 (1994) 233, 1495.

[22] L.N. Savushkin, S. Marcos, M.L. Quelle et al, Phys. Rev. C55 (1997) 167 and references therein.

[23] G. Audi and A.H. Wapstra, Nucl. Phys. A595 (1995) 409. 
[24] P. Möller, J.R. Nix and K.L. Kratz, Atomic data and nuclear data tables 66 (1997), No 2.

[25] P.Bernardos, V.N.Fomenko, N.Van Giai, M.L.Quelle, S.Marcos, R.Niembro and L.N.Savushkin, Phys. Rev. C48 (1993) 2665.

[26] G.Agakichiev et al., Phys. Rev. Lett. 75 (1995) 1272.

[27] G.Chanfray, R.Rapp, and J.Wambach, Phys. Rev. Lett. 76 (1996) 368. 